\section{Effects of visual pattern restriction on sensory reinforcement in the rat'}

\author{
DEVENDRA SINGH, RICHARD J. \\ JOHNSTON, and WILLIAM S. MAKI, JR., \\ North Dakota State University, Fargo, N. D. \\ 58102
}

Effects of early visual pattern experience on sensory reinforcement were studied using both light-onset and light-termination as reinforcers. After weaning, the visual environment of litter-mate rats was restricted for 70 days to either a white or a black and white striped field. Results showed an interaction between early visual pattern experience and type of sensory reinforcer, though light-termination was found to be more effective than light-onset for both early experience groups.

In a previous study (Singh, Johnston, \& Klosterman, 1967) it was shown that rats exposed during early life to an enriched visual pattern gained more body weight, exhibited greater cortical cholinesterase (ChE) activity, and made more lightcontingent bar-pressing responses in a sensory reinforcement test than rats exposed to a restricted visual pattern. The present study was conducted to further investigate the effect of visual pattern experience in early life on sensory reinforcement using both light onset and light termination as reinforcers.

SUBJECTS AND EARLY EXPERIENCE

At 20 days of age, 44 littermate Holtzman female albino rats were randomly assigned to either a visual pattern (VP) group or a restricted visual pattern (RVP) group. The visual environment of Group VP consisted of a board painted with $3 / 4$-in. thick alternating black and white stripes, while the visual environment of the RVP group was restricted to a flat white field (Fig. 1). All Ss were individually housed in stainless steel cages with wire mesh fronts; the visual pattern boards were hung 12 in. in front of the cage racks. All Ss were maintained on ad lib food and water and general rearing conditions were maintained as reported previously (Singh et al, 1967) except all Ss lived in continuous illumination throughout the 70 days of experimental rearing conditions. One rat from the RVP group died during experimental conditions.

\section{APPARATUS}

Two modified operant conditioning chambers $(14 \times 9 \times 10$ in.) were used. Both chambers contained a stationary lever on conditioning (Days 7 and 8) touching the lever terminated the light (light-termination condition) for those Ss which were adapted in a continuously illuminated chamber; for those adapted in a continuously dark chamber, each contact with the lever produced a flash of light (light-onset condition). Number of lever touches were recorded throughout adaptation, conditioning, and extinction during each successive 4-min block.

At the termination of the sensory reinforcement test, all Ss were sacrificed and their brains removed for analysis of ChE activity. The results of this analysis are being reported elsewhere.

\section{RESULTS}

Reported results are based on responses in the first 4-min block only since analysis of data for each 4-min block indicated that significant differences between VP and RVP groups were restricted to the first 4-min block. A repeated-measure analysis of variance was used to analyze the data of 39 Ss (data of five Ss was lost due to equipment failure and death) for last day of adaptation (Day 6), 2 days of conditioning and 3 days of extinction. Significant differences were found for testing conditions $(F=36.48$, $\mathrm{df}=1 / 35, \quad p<.01), \quad$ days $\quad(F=29.36$, $\mathrm{df}=5 / 175, \mathrm{p}<.01)$, and Testing Condition by Days $(F=15.35$, df $=5 / 175, p<.01)$. Rearing condition was not significant as a main effect, but it interacted significantly with testing condition $(F=4.94, d f=1 / 35$, $\mathrm{p}<.05$ ) and Testing Condition by Days $(F=2.45, d f=5 / 175, p<.05)$.

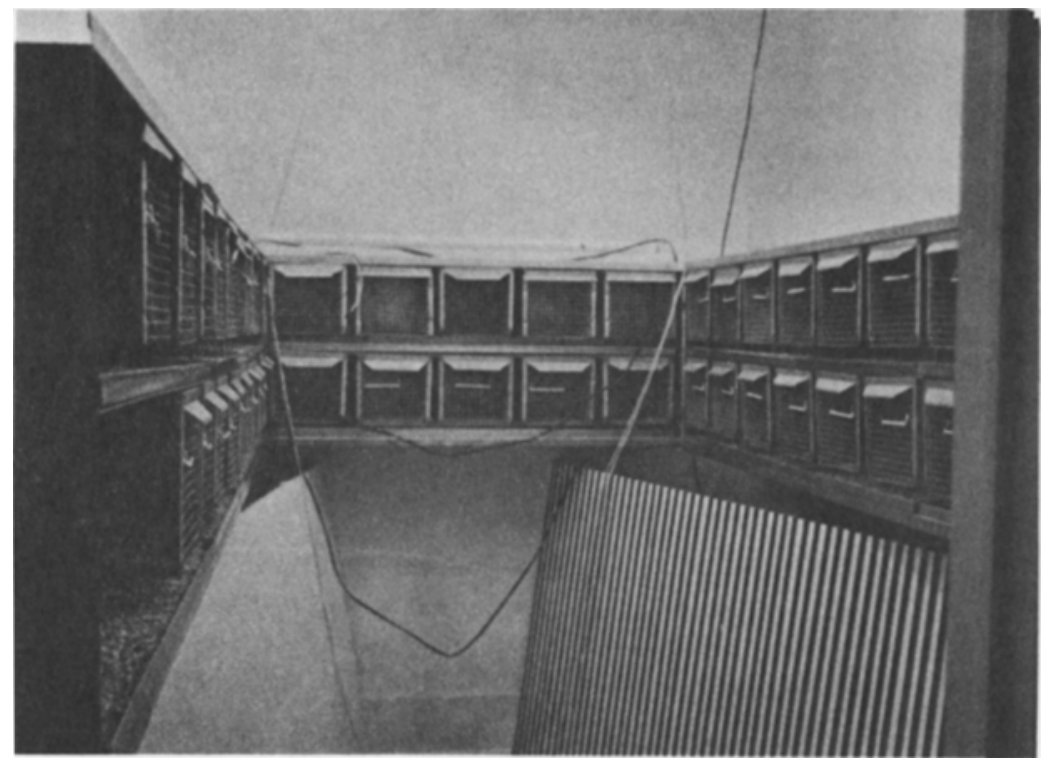

Fig. 1. Rearing room for VP (black and white stripes) and RVP (flat white) groups. Visual pattern boards were suspended in front of each rack of cages. 


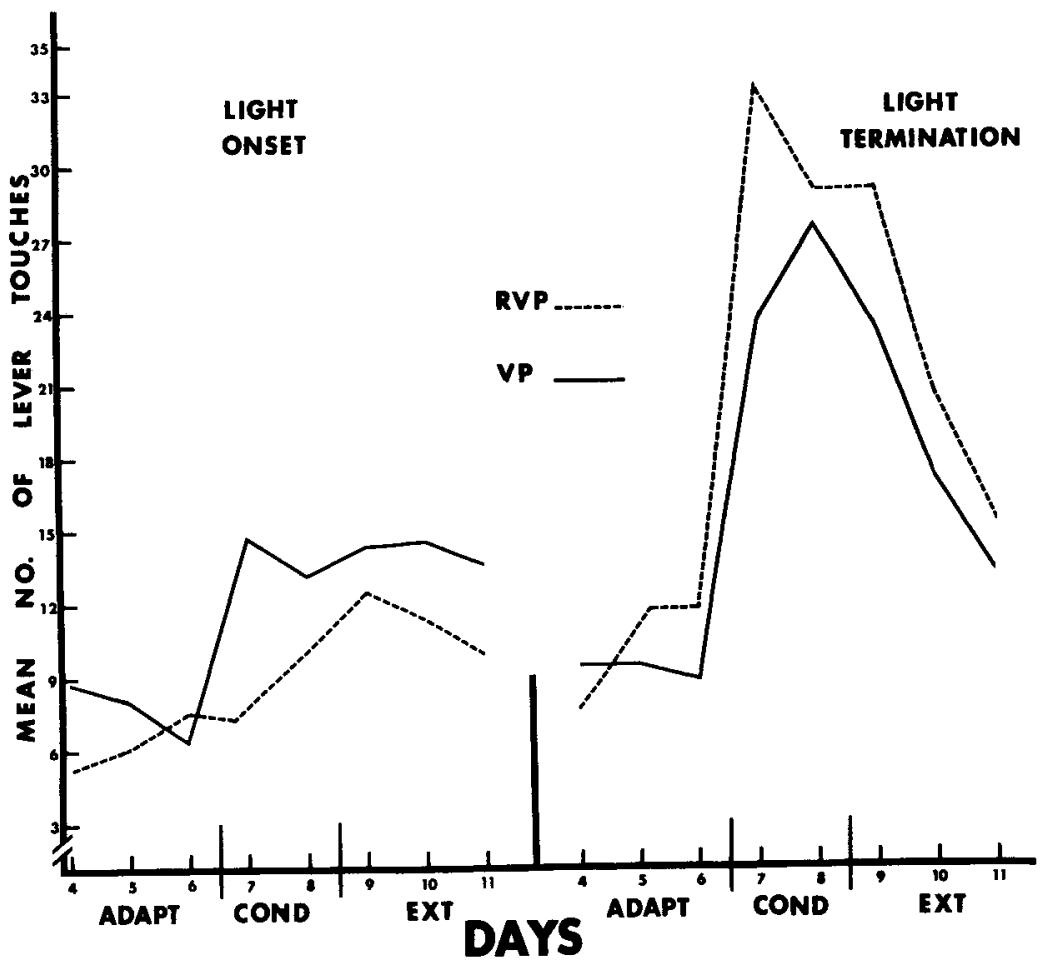

Fig. 2. Mean number of lever touches during the last three adaptation days, conditioning, and extinction for both VP and RVP groups under both light onset and light termination.

Figure 2 presents the mean number of lever touches for both the RVP and VP groups for the last 3 days of adaptation, 2 days of conditioning, and 3 days of extinction under both light-onset and light-termination conditions. As evident from the figure, both groups made more responses when light termination was used as reinforcer than when light onset was used. Furthermore, while VP, compared to the RVP group, made more responses in the conditioning and extinction days under the light-onset condition, this trend is completely reversed under the light-termination condition. The significantly better performance $(p<.05)$ of the VP group for the first day of conditioning under the light-onset condition than the RVP group is consistent with the previous findings of Singh et al (1967). However, under light termination, the RVP group made significantly more conditioning. Under both light-onset and light-termination conditions, the VP and RVP groups did not differ significantly from each other on the second day (Day 8) of conditioning or any of the extinction days.

$$
\text { DISCUSSION }
$$

In general, results show that early visual pattern experience affects sensory reinforcement performance whether light onset or light termination is used as a reinforcer. However, contrary to the findings of Barnes \& Kish (1957), Hurwitz (1956), and Robinson (1959), light termination was found to be a more effective reinforcer than light onset for both RVP and VP groups. The greater effectiveness of light termination as a reinforcer could not be due to the aversive properties of light alone; if this were the case, no sensory conditioning should have taken place under light-onset conditions.
The better performance by both RVP and VP groups under light-termination conditions could probably be explained by referring to the differential amounts of darkness experienced by the light-onset and light-termination groups during adaptation period. Specifically, Ss that worked for light termination were adapted for 6 days in a continuously lighted chamber while Ss that worked for light onset were adapted in a dark chamber for 6 days. Thus, when conditioning was initiated, the light-onset groups had experienced both light and darkness while the light-termination groups had never experienced darkness. It is, therefore, possible that the reinforcer (darkness) in the light-termination condition was more effective due to its novelty than the reinforcer in the light-onset condition.

The most difficult finding to explain is the performance of the VP group. When light onset was used, Group VP made significantly more responses than Group RVP which is consistent with the findings of Singh et al (1967); however, when light termination was used, Group VP made significantly fewer responses than the RVP group. The authors have no explanation for the performance of the VP group under light-onset vs light-termination conditions, except to point out the interaction between testing situations and early visual pattern experience.

\section{REFERENCES}

BARNES, G. W., \& KISH, G. B. Behavioral effects of the cessations of weak light energy. American Psychologist, 1957, 12,411 (abstract). reinforced by light. British Journal of Animal Behaviour, 1956, 4, 31-33.

ROBINSON, J. S. Light onset and termination as reinforcers for rats living under normal light conditions. Psychological Reports, 1959, 5, 793-796.

SINGH, D., JOHNSTON, R. J., \& KLOSTERMAN, H. J. Effect on brain enzyme and behaviour in the rat of visual pattern restriction in early life. Nature, 1967, 216, 1337-1338.

\section{NOTE}

1. This study was supported by funds from The Graduate School, North Dakota State University.
HURWITZ, H. M. B.Conditioned responses in rats 